\title{
Endosonography today: An overview
}

\author{
Showkat Bashir \\ Division of Gastroenterology, Hepatology and Nutrition, George Washington University, NW Washington, DC 20037, USA
}

$\begin{array}{ll}\text { Abstract } & \begin{array}{l}\text { Over the past two decades, the combination of gastrointestinal endoscopy and ultrasonography } \\ \text { has shown remarkable progress. This progress has allowed endoscopists to diagnose and } \\ \text { treat broad range of not only gastrointestinal disorders but also mediastinal, pancreatic, and } \\ \text { perirectal diseases. Endoscopic ultrasound (EUS) offers a vital complementary role to traditional } \\ \text { cross-sectional imaging. EUS helps to obtain tissue samples under real-time ultrasonographic } \\ \text { guidance using fine-needle aspiration EUS. The ever expanding use of EUS makes it imperative } \\ \text { for primary care providers to be familiar with this technology and its application. This progress } \\ \text { has made EUS an integral and necessary part of any good endoscopy unit and is here to stay. }\end{array} \\ \text { Key words } & \begin{array}{l}\text { Endoscopic, gastrointestinal, ultrasound } \\ \text { Kand }\end{array}\end{array}$

\section{Introduction}

Endoscopic ultrasound (EUS) is an effective and safe method for imaging the gastrointestinal wall and surrounding structures. EUS can image the posterior mediastinum, pancreas, retroperitoneum, biliary tree, and the perirectal space. The EUS was developed in the early 1980s. ${ }^{[1]}$ The pioneers developed images, defined anatomy, correlated with computed tomography (CT) images, and operative findings. However, the instruments were bulky, mechanical, and cumbersome to use. In addition, image resolution was poor which limited its widespread use. Steady progress in research and development now has provided high-resolution image processing, flexible equipment with capacity for fine-needle aspiration (FNA).

For a quality EUS, the endoscopist's skills and training need to be more advanced than with routine endoscopic procedures. In the United States, most EUS training is limited to $4^{\text {th }}$ year of gastroenterology fellowship and require a certain number of procedures for certification. ${ }^{[2]}$ In addition to skills, one must

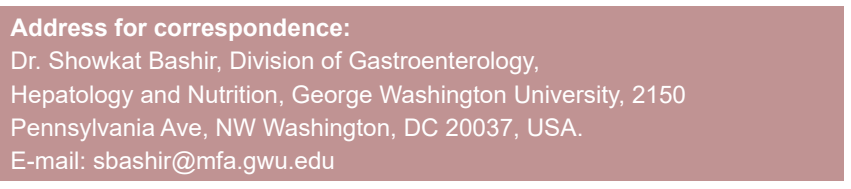

\begin{tabular}{|l|c|}
\hline \multicolumn{2}{|c|}{ Access this article online } \\
\hline \multirow{2}{*}{ Website: } & Quick Response Code \\
\hline www.jeonline.in & \\
\hline DOI: & \\
10.4103/0976-5042.193736 & D \\
\hline
\end{tabular}

be familiar with the anatomy surrounding the gastrointestinal tract. Particular emphasis should be placed on vascular landmarks including aorta, its major branches, and the mesenteric venous system. These anatomic constants provide a valuable guide during EUS.

During EUS a detailed image of the normal and pathological process can be obtained. The method can be used both for primary diagnosis and in the follow-up of disorders. It improves characterization, enhances staging of neoplasms of the gastrointestinal tract, and surrounding organs. It also aids in detecting small lesions, which cannot be seen with other imaging modalities.

\section{Basic Principles of Ultrasound}

Sound waves are generated by the ultrasound transducer and emitted into surrounding tissues. These emitted sound waves reflect off tissues and return to the transducer, allowing the time elapsed to be translated into distances from the transducer. Furthermore, variable penetration of sound through different tissue structures allows characteristics to be mapped visually using bright or dark spots on a monitor. The ultrasound representation of fat is bright or white, and fluid is dark or

This is an open access article distributed under the terms of the Creative Commons Attribution-NonCommercial-ShareAlike 3.0 License, which allows others to remix, tweak, and build upon the work non-commercially, as long as the author is credited and the new creations are licensed under the identical terms.

For reprints contact: reprints@medknow.com

How to cite this article: Bashir S. Endosonography today: An overview. J Dig Endosc 2016;7:98-103. 
black. Parenchymal organs characteristics are intermediate or gray. Nearly, every parenchymal organ has unique ultrasound imaging features, making them recognizable to the practiced eye.

\section{Types of Echoendoscopes and Probes}

During EUS a specialized endoscope called echoendoscope is used. The echoendoscopes differ from normal endoscopes by having a sophisticated ultrasound transmission and reception capability at the tip of the instrument. The two types of echoendoscopes are radial [Figure 1a] and linear [Figure 1b]. The linear instruments scan in the same plane as the long axis of the endoscope, whereas mechanical radial instruments have a rotating mechanical ultrasound probe that scans in a circle at $90^{\circ}$ to the long axis of the echoendoscope. The ultrasound signal is then integrated by a complex processor and transmitted in real-time to a video screen. The curved linear array echoendoscope is optimal for performing EUS-FNA since it permits real-time visualization of the needle as it is advanced into the lesion.

Besides the echoendoscope, high-frequency probes are also available for imaging. These probes can be passed through the working channels of the regular endoscopes into the gut lumen, common bile, and pancreatic duct. These probes have enhanced imaging of the gut wall lesions, especially small lesion and intraductal pathology of the common bile duct and pancreatic duct.

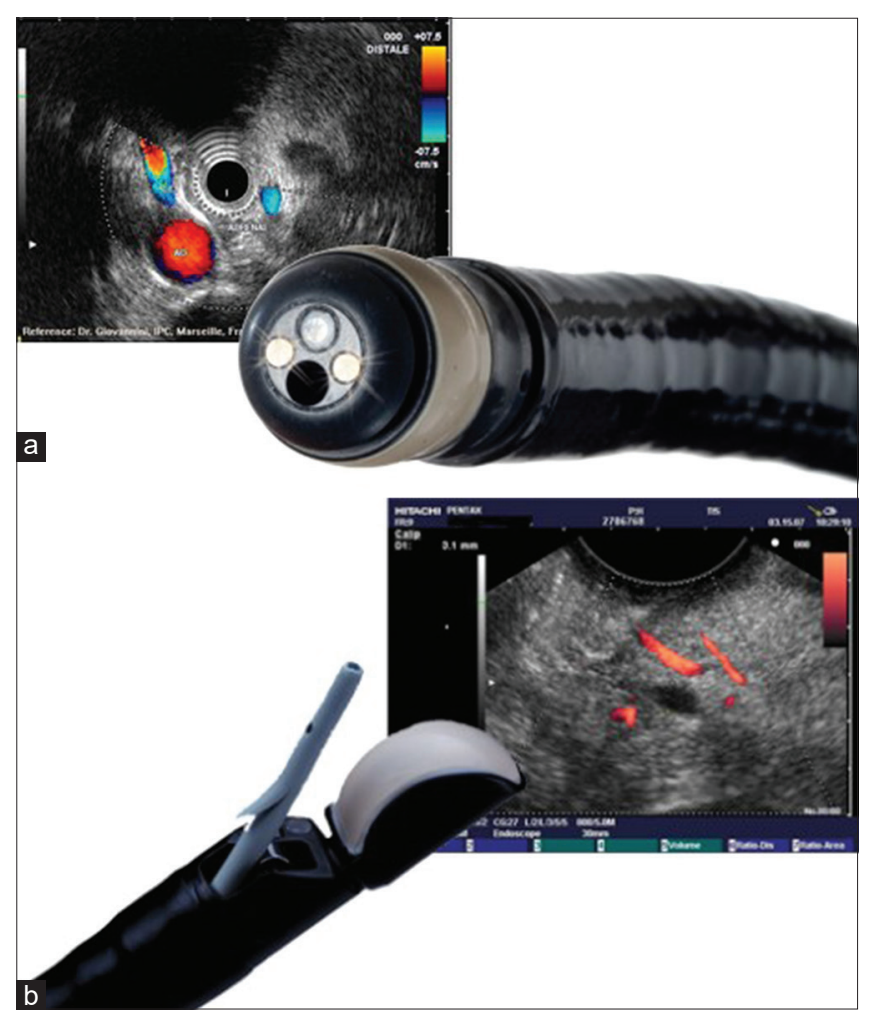

Figure 1: Two types of echoendoscopes. (a) Radial echoendoscope, (b) linear echoendoscope. (Images reproduced after permission from Pentax of America.)

\section{Patient Preparation}

The patient preparation for upper EUS is no different from standard upper endoscopy. The patient needs to remain fasting for at least $8 \mathrm{~h}$ before the procedure as sedation is required. If intervention like cyst drainage or FNA is planned it is imperative to use antibiotics either before or in the immediate postprocedure period. For solid tumors of the pancreas and other organs FNA can be done safely without antibiotic prophylaxis. The use of anticoagulation and antiplatelet medication needs to be addressed before performing EUS, especially for EUS-FNA.

Endorectal EUS can be performed after preparation with enemas. However, for proximal colonic lesions and if any therapeutic intervention is planned standard colonoscopy preparation is needed.

\section{Endoscopic Ultrasound and Wall Layers of Gastrointestinal Tract}

The wall layers of the gastrointestinal wall include mucosa, lamina propria, muscularis mucosae, submucosa, muscularis propria, and serosa. The interface between luminal fluid, mucosa, and other components of the wall layer produce echo on EUS are illustrated in Figure 2a and $b$.

\section{Endoscopic Ultrasound in Mediastinum and Lung Cancer}

EUS and EUS-FNA can be used to diagnose benign and malignant disease in the mediastinum. In benign reactive lymphadenopathy and the granulomatous diseases (e.g. sarcoidosis, tuberculosis, and histoplasmosis) both EUS and EUS-FNA offer useful information and tissue diagnosis. It is a safe and cost-effective alternative to other diagnostic methods (e.g., mediastinoscopy) and has a high diagnostic yield in such patients. ${ }^{[3,4]}$

In lung cancer and other malignancy associated lymphadenopathy in the posterior or inferior mediastinum,

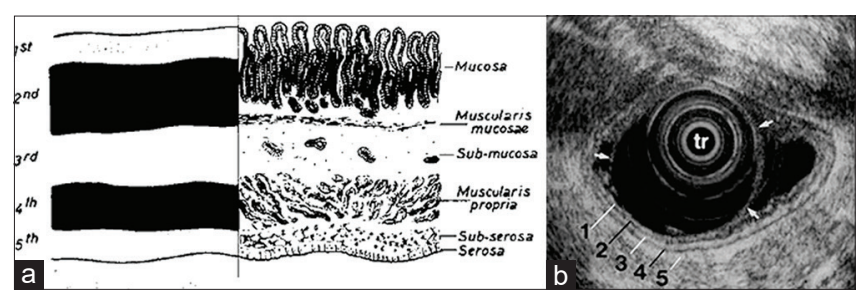

Figure 2: ( $a$ and b) Correspondence between the endoscopic ultrasound and anatomic layers of the normal wall. (1) Interface between stomach fluid and superficial mucosa, (2) lamina propria and muscularis mucosae, (3) submucosa and interface between submucosa and muscularis propria, (4) muscularis propria and (5) interface between serosa and surrounding tissue. (Adapted after permission from Gastrointestinal Endoscopy Clinics of North America, October 1992, Volume 2, Number 4, Page 604, Figure 2) 
EUS-FNA is particularly helpful [Figure 3]. The paratracheal, aortopulmonary, subcarinal, paraesophageal, and hilar lymphadenopathy in the setting of lung cancer make a sampling of these nodes important. The sampling with EUS-FNA of these nodes remains minimally invasive and can avoid surgical mediastinoscopy. Besides, avoiding thoracotomy or thoracoscopy, EUS provided a cost-effective strategy for staging in lung cancer. In lung cancer, EUS-FNA allows sampling of the celiac lymph nodes, left adrenal gland, and left lobe of the liver allowing accurate staging. ${ }^{[5-8]}$

\section{Endoscopic Ultrasound in the Benign Upper Gastrointestinal Tract Lesions}

A subepithelial mass or a bulge encountered during an endoscopy can arise from within any layer of the gastrointestinal tract wall. The mass could be intramural or extramural. Such lesions are usually found incidentally (incidentalomas) during endoscopy. The differential diagnosis includes a number of benign and malignant nonepithelial gastric wall tumors, intramural vessels, and extrinsic compression from extramural structures. Endoscopy alone cannot accurately distinguish between intramural and extramural lesions. On the other hand, EUS and FNA have provided a major breakthrough for better characterization of such masses. ${ }^{[9-11]}$

The commonly encountered subepithelial lesions of the upper gastrointestinal tract are gastrointestinal stromal tumors [Figure 4a-c], lipomas [Figure 5a and b], carcinoids [Figure $6 \mathrm{a}$ and $\mathrm{b}$ ], pancreatic rests [Figure $7 \mathrm{a}$ and $\mathrm{b}$ ], and varices [Figure $8 \mathrm{a}$ and $\mathrm{b}$ ]. In addition, duplication cysts, granular cell tumors, and leiomyomas are easily identifiable on EUS. The ability of EUS to determine the size, echo features, wall layer origin, and sampling with FNA had greatly helped in differentiating these lesions. ${ }^{[12,13]}$

\section{Endoscopic Ultrasound in Malignant Upper Gastrointestinal Tract Lesions}

In esophageal cancer, the prognosis is strongly associated with the stage of cancer. EUS accurately assesses the tumor (T) and nodal (N) stage and allows appropriate treatment. In addition, EUS also remains useful for detecting disease recurrence. Once initial CT and positron emission tomography scan show no diffuse distant metastasis from primary esophageal neoplasm EUS demonstrates higher than $95 \%$ accuracy in evaluating celiac and periesophageal lymphadenopathy (N-stage) and wall layer (T-stage) involvement. ${ }^{[14-16]}$

For early esophageal cancers limited to mucosa and superficial submucosa, EUS plays a pivotal role. In such cases, endoscopic mucosal resection can be performed without the morbidity and mortality associated with esophagectomy. ${ }^{[17,18]}$

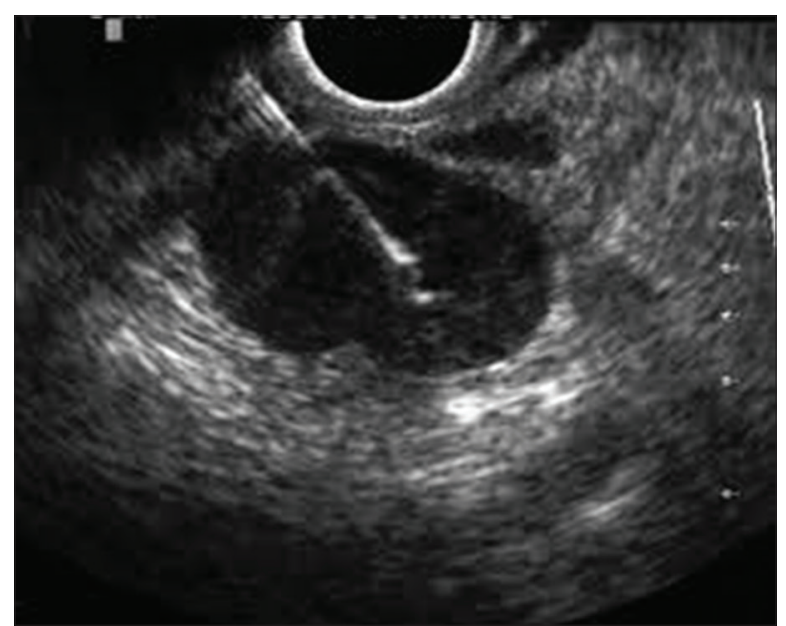

Figure 3: Endoscopic ultrasound fine-needle aspiration of a sub-carinal lymph node in a patient with bulky mediastinal lymphadenopathy from metastatic ovarian cancer

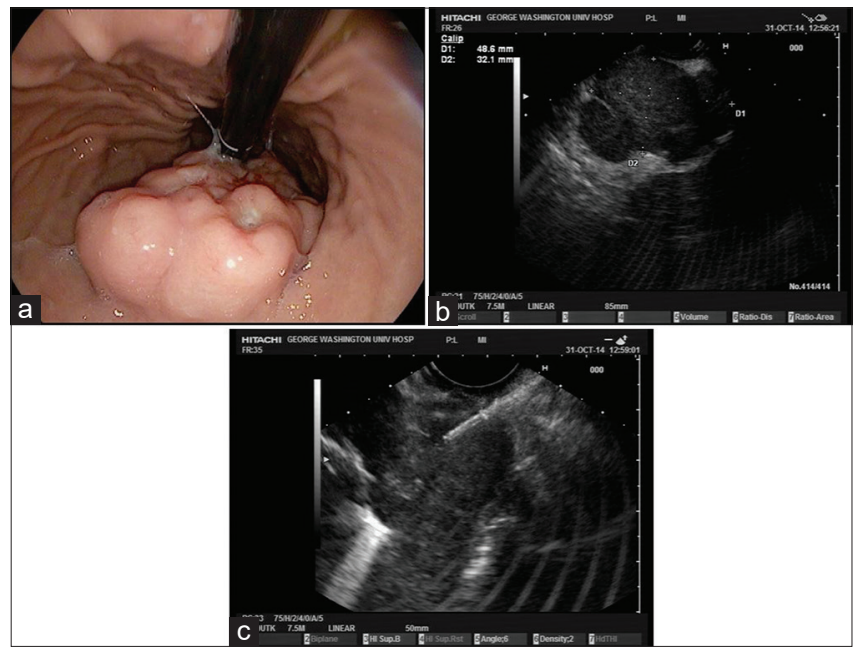

Figure 4: (a-c) Ulcerated gastric subepithelial mass noted as hypoechoic mass on endoscopic ultrasound arising from muscularis propria. Fine needle aspiration under endoscopic ultrasound guidance confirmed the diagnosis of gastrointestinal stromal tumor.

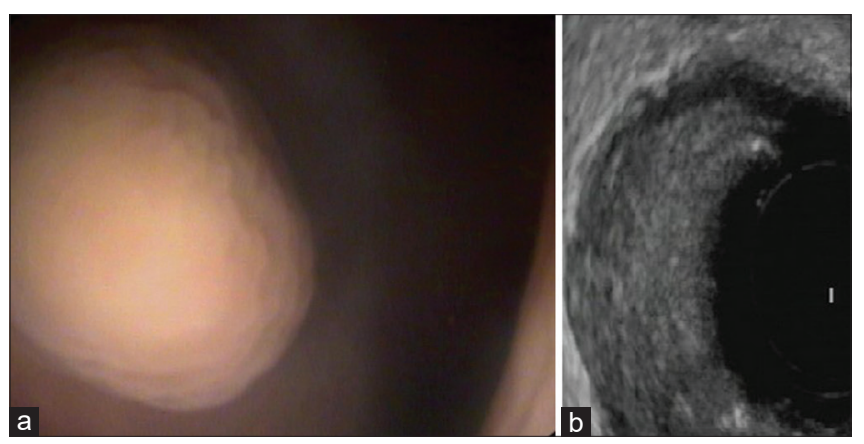

Figure 5: ( $a$ and $b)$ Soft descending duodenal lesion on endoscopy which on Endoscopic ultrasound demonstrated a homogeneous hyperechoic submucosal lesion consistent with a benign lipoma

In mucosa-associated lymphoid tissue lymphoma of the stomach, EUS determines the wall layer involvement and local 
regional lymphadenopathy. Limited wall layer involvement with no regional lymphadenopathy in these patients suggests early stage disease which responds to Helicobacter pylori eradication and avoids unnecessary chemotherapy. ${ }^{[19]}$

The role of EUS in gastric adenocarcinoma in the United States remains limited as most cases are diagnosed at advanced stages only and cross-sectional imaging remains standard to evaluate for local and metastatic disease.

\section{Endoscopic Ultrasound and Pancreatic Cancer}

The pancreatic cancer is one of the common causes of cancer-related death in the US. EUS is useful in the staging and tissue diagnosis of pancreatic cancer. The goal of preoperative staging is to identify patients with resectable disease who might benefit from surgery while avoiding surgery in patients with locally advanced or metastatic disease. ${ }^{[20]}$

The staging patients with pancreatic cancer include determining the tumor-node-metastasis (TNM) stage as well as evaluating for vascular invasion. Pancreatic cancer is staged using the TNM system. EUS is useful for tumor [Figure 9] and node staging [Figure 10], while imaging modalities, such as CT or magnetic resonance imaging are required for metastasis. In general, EUS is more accurate than CT for T-staging of smaller tumors, whereas CT is more accurate than EUS for larger tumors. EUS also appears to be as accurate as CT for Nodal
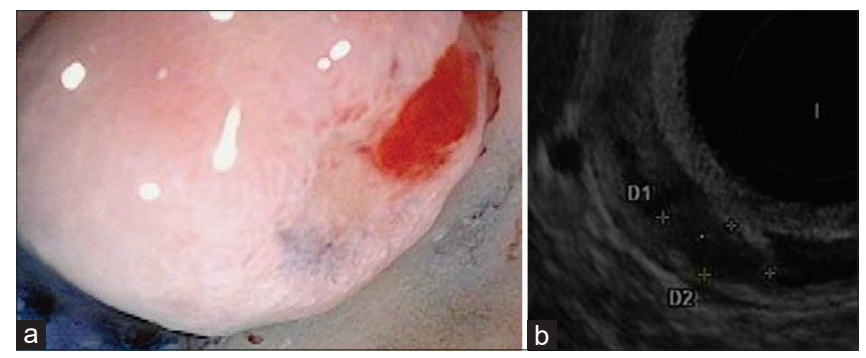

Figure 6: ( $a$ and b) Duodenal bulb carcinoid tumor as a hypoechoic lesion within the submucosa on endoscopic ultrasound with intact muscularis layer allowing for endoscopic mucosal resection

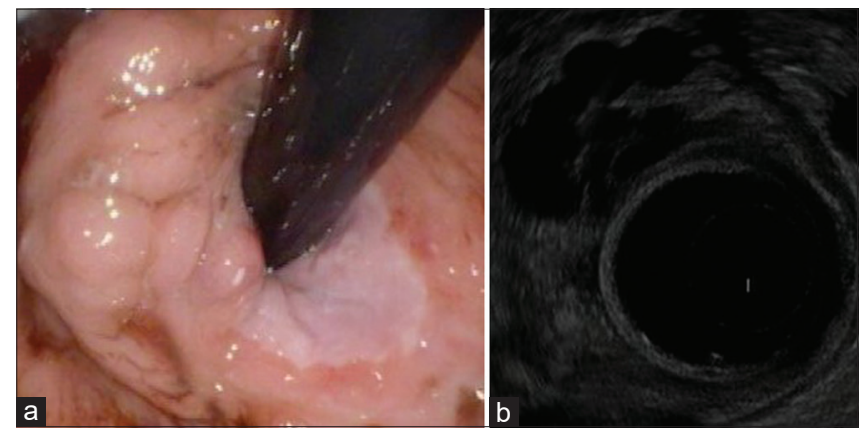

Figure 8: ( $a$ and $b)$ Gastric fundal varices noted as enlarged folds and subepithelial prominences on endoscopy and on endoscopic ultrasound as anechoic structures within the submucosa staging. ${ }^{[21]}$ The presence or absence of vascular invasion is a major determinant of tumor resectability. However, vascular invasion does not automatically preclude resection since some involved vessels can be resected and reconstructed. Thus, it is important to know whether vascular invasion is present, and if it is, to determine the specific anatomic location and the degree of vascular invasion. The reported accuracy of EUS for TNM staging and for determining vascular invasion is highly variable among studies. ${ }^{[22]}$

EUS-FNA is technically successful in $90 \%-95 \%$ of procedures, with high sensitivity and specificity. In pancreatic cancer, a meta-analysis of 15 studies with 1860 patients found that overall, the sensitivity of EUS-FNA for pancreatic cancer was $92 \%$, and the specificity was $96 \%$. In one report, the presence of a cytopathologist in attendance for all aspiration procedures was associated with a high degree of accuracy (95\%) in diagnosing pancreatic cancer. ${ }^{[23]}$

\section{Endoscopic Ultrasound and Rectal Cancer}

Endorectal ultrasound offers excellent tumor (T) staging in rectal cancer. Many centers use rectal EUS preoperatively on routine basis in rectal cancer. Compared with cross-sectional imaging rectal EUS frequently upstages patients, making them eligible for neoadjuvant treatment. ${ }^{[24]}$ FNA should be reserved

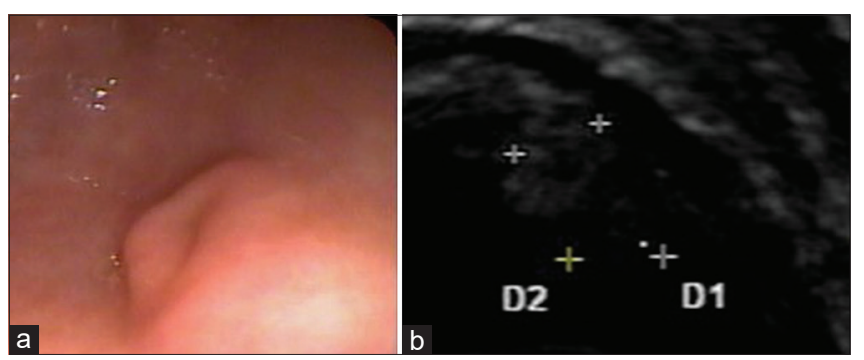

Figure 7: $(a$ and $b)$ Umbilical antral lesion noted as heterogeneous lesion within the gastric submucosa, features of ectopic pancreatic rest

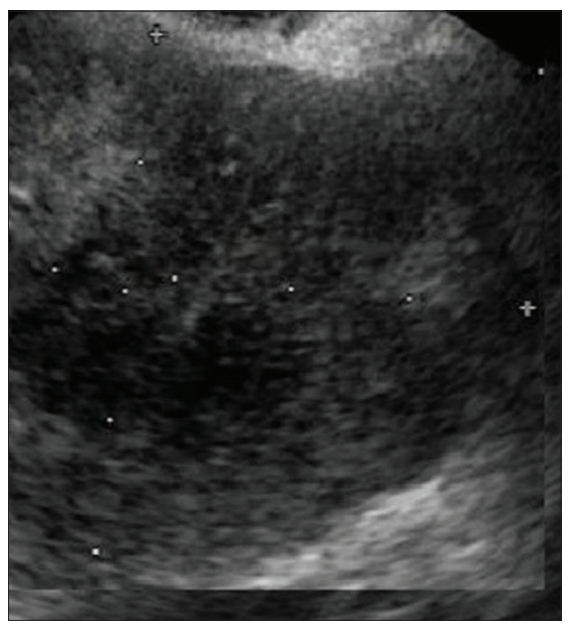

Figure 9: Pancreatic body mass on endoscopic ultrasound 
for patients who appear to have the early T-stage disease but with suspicious peri-iliac lymphadenopathy. The accurate staging ability of rectal EUS and FNA with appropriate preoperative chemoradiation in those patients with transmural disease and/or lymphadenopathy translates into improved outcomes in terms of reduced recurrence rates, decreased the frequency of colostomy, and reduction in treatment complications. However, no improvement in survival is apparent. ${ }^{[25]}$ The role of EUS in patients with recurrent rectal cancer and as follow-up after resection remains unsettled.

\section{Endoscopic Ultrasound in Chronic Pancreatitis}

EUS offers valuable information in benign disease of the bile duct and pancreas. The close proximity of the pancreas and bile duct to the gastroduodenal wall allows for high-resolution imaging. In addition, EUS is less invasive than endoscopic retrograde cholangiopancreatography for imaging the pancreato-biliary system.

The imaging with EUS images the parenchyma and the ductal system of the pancreas. The normal pancreas parenchyma is seen as homogenous, fine, granular, salt and pepper appearance throughout with smooth margins. ${ }^{[26]}$ The main pancreatic duct is noted as anechoic structure to have average diameter $1.5-2.4 \mathrm{~mm}$ in healthy patients. In patients with chronic pancreatitis EUS is helpful in assessing quantitative and qualitative criteria in both parenchyma and ductal symptoms. The commonly used diagnostic criteria for chronic pancreatitis include parenchymal and ductal changes. The parenchymal changes can be variable and include diffuse or localized lobulations, hyperechoic streaky calcifications, punctate calcifications, chronic pseudocysts, atrophy, and irregular margins. On the other hand, the ductal changes can vary between hyperechoic ductal wall, dilated ducts, clubbed or ectatic ductal side walls [Figure 11]. These changes are well elucidated in Rosemont classification with scoring system which is beyond the scope of this review. ${ }^{[2]]}$

\section{Endoscopic Ultrasound in Biliary Disease}

About $20 \%$ of patients with gallstone can have common bile duct stones. For diagnosis of common bile duct stones usually, noninvasive imaging like transabdominal ultrasound, CT, magnetic resonance cholangiopancreatography is utilized with relatively good sensitivity of about $90 \%$. However, in some patients like those with recurrent idiopathic pancreatitis small common duct stones or gallbladder sludge may be missed by these imaging modalities. EUS offers a very safe imaging for diagnosis of biliary sludge within the gallbladder or the bile duct with both sensitivity and specificity more than $90 \%{ }^{[28]}$ In uncomplicated benign bile duct disorders such as choledochal cysts, EUS provides the size, contents, and wall delineation of such cysts [Figure 12].

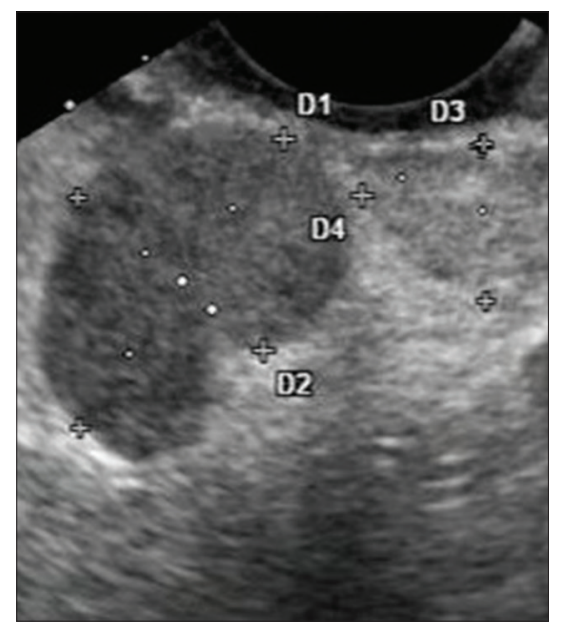

Figure 10: Celiac lymphadenopathy on endoscopic ultrasound
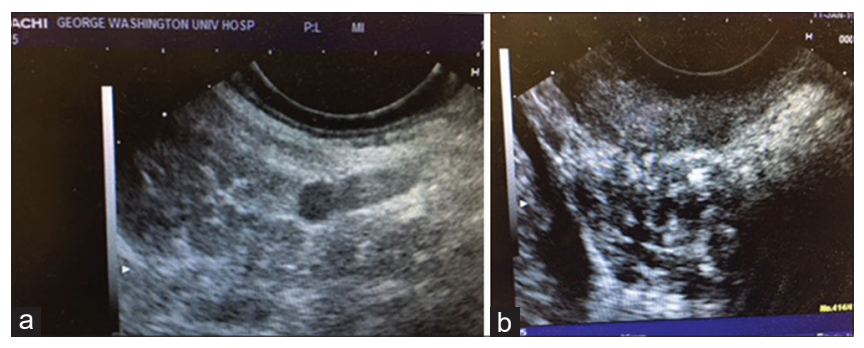

Figure 11: ( $a$ and $b$ ) Endoscopic ultrasound findings of chronic pancreatitis (lobulations, ductal dilation, hyperechoic ductal walls, and punctate parenchymal calcifications)

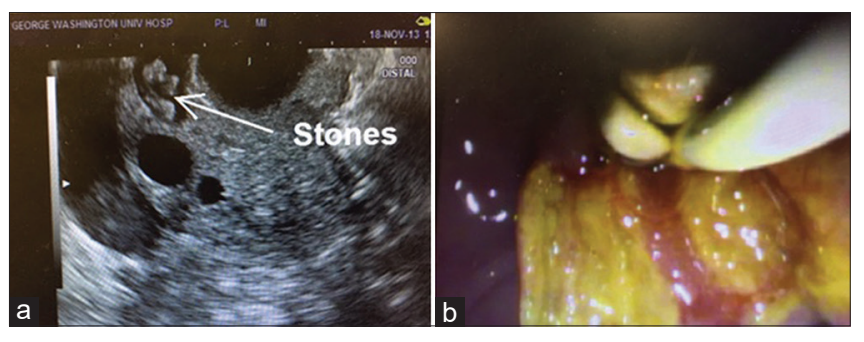

Figure 12: (a and b) Common bile duct stones on endoscopic ultrasound followed by extraction using endoscopic retrograde cholangiopancreatography

\section{Conclusion}

EUS and EUS-FNA are in widespread use for investigation of subepithelial lesions of the gastrointestinal tract and staging of pancreas, lung, mediastinal, and perirectal tumors. This modality is safe, cost-effective, and accurate. It also compliments cross-sectional imaging for all these tumors. Due to widespread use and multiple indications, the primary care physicians need to familiarize themselves with indications for EUS and EUS-FNA.

\section{Financial support and sponsorship Nil.}

\section{Conflicts of interest}

There are no conflicts of interest. 


\section{References}

1. Dimagno EP, Regan PT, Clain JE, James EM, Buxton JL. Human endoscopic ultrasonography. Gastroenterology 1982;83:824-9.

2. EUS Core Curriculum. Communication from the ASGE training committee. Gastrointest Endosc 2012;76:476-81.

3. ASGE Standards of Practice Committee, Jue TL, Sharaf RN, Appalaneni V, Anderson MA, Ben-Menachem T, et al. Role of EUS for the evaluation of mediastinal adenopathy. Gastrointest Endosc 2011;74:239-45.

4. Fritscher-Ravens A, Sriram PV, Topalidis T, Hauber HP, Meyer A, Soehendra N, et al. Diagnosing sarcoidosis using endosonography-guided fine-needle aspiration. Chest 2000;118:928-35.

5. Hawes RH, Gress F, Kesler KA, Cummings OW, Conces DJ Jr. Endoscopic ultrasound versus computed tomography in the evaluation of the mediastinum in patients with non-small-cell lung cancer. Endoscopy 1994;26:784-7.

6. Uemura S, Yasuda I, Kato T, Doi S, Kawaguchi J, Yamauchi T, et al. Preoperative routine evaluation of bilateral adrenal glands by endoscopic ultrasound and fine-needle aspiration in patients with potentially resectable lung cancer. Endoscopy 2013;45:195-201.

7. Kramer H, Groen HJ. Diagnosis of mediastinal and left adrenal abnormalities with endoscopic ultrasonography. Respir Med 2005;99:926-8.

8. Larsen SS, Vilmann P, Krasnik M, Dirksen A, Clementsen P, Maltbaek N, et al. Endoscopic ultrasound guided biopsy performed routinely in lung cancer staging spares futile thoracotomies: Preliminary results from a randomised clinical trial. Lung Cancer 2005;49:377-85.

9. Hwang JH, Saunders MD, Rulyak SJ, Shaw S, Nietsch H, Kimmey MB. A prospective study comparing endoscopy and EUS in the evaluation of GI subepithelial masses. Gastrointest Endosc 2005;62:202-8.

10. Watson RR, Binmoeller KF, Hamerski CM, Shergill AK, Shaw RE, Jaffee IM, et al. Yield and performance characteristics of endoscopic ultrasound-guided fine needle aspiration for diagnosing upper GI tract stromal tumors. Dig Dis Sci 2011;56:1757-62.

11. Savides TJ. Endoscopic ultrasonography. In: Gress F, editors. Gastrointestinal Submucosal Masses. Blackwell Science; 2001.

12. Hunt GC, Smith PP, Faigel DO. Yield of tissue sampling for submucosal lesions evaluated by EUS. Gastrointest Endosc 2003;57:68-72.

13. American Gastroenterological Association Institute. American Gastroenterological Association Institute medical position statement on the management of gastric subepithelial masses. Gastroenterology 2006;130:2215-6.

14. Tio TL, Coene PP, den Hartog Jager FC, Tytgat GN. Preoperative TNM classification of esophageal carcinoma by endosonography. Hepatogastroenterology 1990;37:376-81.
15. Vilgrain V, Mompoint D, Palazzo L, Menu Y, Gayet B, Ollier P, et al. Staging of esophageal carcinoma: Comparison of results with endoscopic sonography and CT. AJR Am J Roentgenol 1990;155:277-81.

16. Lowe VJ, Booya F, Fletcher JG, Nathan M, Jensen E, Mullan B, et al. Comparison of positron emission tomography, computed tomography, and endoscopic ultrasound in the initial staging of patients with esophageal cancer. Mol Imaging Biol 2005;7:422-30.

17. Hasegawa N, Niwa Y, Arisawa T, Hase S, Goto H, Hayakawa T. Preoperative staging of superficial esophageal carcinoma: Comparison of an ultrasound probe and standard endoscopic ultrasonography. Gastrointest Endosc 1996;44:388-93.

18. Rice TW, Blackstone EH, Adelstein DJ, Zuccaro G Jr., Vargo JJ, Goldblum JR, et al. Role of clinically determined depth of tumor invasion in the treatment of esophageal carcinoma. J Thorac Cardiovasc Surg 2003; 125:1091-102.

19. Fischbach W, Al-Taie O. Staging role of EUS. Best Pract Res Clin Gastroenterol 2010;24:13-7.

20. Ahmad NA, Lewis JD, Ginsberg GG, Rosato EF, Morris JB, Kochman ML. EUS in preoperative staging of pancreatic cancer. Gastrointest Endosc 2000;52:463-8.

21. Hunt GC, Faigel DO. Assessment of EUS for diagnosing, staging, and determining resectability of pancreatic cancer: A review. Gastrointest Endosc 2002;55:232-7.

22. Puli SR, Singh S, Hagedorn CH, Reddy J, Olyaee M. Diagnostic accuracy of EUS for vascular invasion in pancreatic and periampullary cancers: A meta-analysis and systematic review. Gastrointest Endosc 2007;65:788-97.

23. Harewood GC, Wiersema MJ. Endosonography-guided fine needle aspiration biopsy in the evaluation of pancreatic masses. Am J Gastroenterol 2002;97:1386-91.

24. Guinet C, Buy JN, Ghossain MA, Sézeur A, Mallet A, Bigot JM, et al. Comparison of magnetic resonance imaging and computed tomography in the preoperative staging of rectal cancer. Arch Surg 1990;125:385-8.

25. Mukae M, Kobayashi K, Sada M, Yokoyama K, Koizumi W, Saegusa M. Diagnostic performance of EUS for evaluating the invasion depth of early colorectal cancers. Gastrointest Endosc 2015;81:682-90.

26. Nattermann C, Goldschmidt AJ, Dancygier H. Endosonography in chronic pancreatitis - A comparison between endoscopic retrograde pancreatography and endoscopic ultrasonography. Endoscopy 1993;25:565-70.

27. Catalano MF, Sahai A, Levy M, Romagnuolo J, Wiersema M, Brugge W, et al. EUS-based criteria for the diagnosis of chronic pancreatitis: The Rosemont classification. Gastrointest Endosc 2009;69:1251-61.

28. Garrow D, Miller S, Sinha D, Conway J, Hoffman BJ, Hawes RH, et al. Endoscopic ultrasound: A meta-analysis of test performance in suspected biliary obstruction. Clin Gastroenterol Hepatol 2007;5:616-23. 